\title{
Whole extracts of Radix Achyranthis Bidentatae and Radix Cyathulae promote angiogenesis in human umbilical vein endothelial cells in vitro and in zebrafish in vivo
}

\author{
XUELIN ZHOU ${ }^{1-3}$, WING-SUM SIU ${ }^{1-3}$, CHENG ZHANG $^{1,2}$, CHEUK-LUN LIU $^{1,2}$, LING CHENG $^{1,2}$, \\ HIN-FAI KWOK ${ }^{1,2}$, CHAK-HEI FUNG ${ }^{1-3}$, JACQUELINE CHOR-WING TAM ${ }^{1,2}$, CHING-PO LAU $^{1,2}$, \\ CLARA BIK-SAN LAU ${ }^{1-3}$, PING-CHUNG LEUNG ${ }^{1-4}$, LEUNG-KIM HUNG ${ }^{4}$ and CHUN-HAY KO ${ }^{1-3}$
}

\begin{abstract}
${ }^{1}$ Institute of Chinese Medicine; ${ }^{2}$ State Key Laboratory of Phytochemistry and Plant Resources in West China, Shatin, The Chinese University of Hong Kong, Shatin, New Territories; ${ }^{3}$ Shenzhen Research Institute, The Chinese University of Hong Kong, Shenzhen, Guangdong; ${ }^{4}$ Department of Orthopaedics and Traumatology, The Chinese University of Hong Kong, Shatin, New Territories, Hong Kong SAR, P.R. China
\end{abstract}

Received August 20, 2015; Accepted November 4, 2016

DOI: $10.3892 /$ etm.2017.4053

\begin{abstract}
Although Radix Achyranthis Bidentatae (RAB) and Radix Cyathulae (RC) are from two different medicinal plants, they are both used as 'Niu-Xi', a widely used traditional Chinese medicine that is believed to stimulate menstruation and affect bone injury. Angiogenesis is actively involved in treating these illnesses. The aim of the present study was to investigate whether the whole extracts of RAB and RC possess pro-angiogenic effects. In order to examine this idea whole extracts of $\mathrm{RAB}$ and $\mathrm{RC}$ were extracted with boiling water followed by ethanol, respectively. Results from the MTT, wound healing and tube formation assays in human umbilical vein endothelial cells (HUVECs) in vitro revealed that the whole extracts of RAB and RC did not increase cell proliferation or tube formation, but enhanced cell migration. Their angiogenic effects were also confirmed in zebrafish in vivo via increasing the sprout numbers in the sub-intestinal vessel. As determined by quantitative polymerase chain reaction, the whole extracts of RAB and RC both regulated the expression of cell migration-related genes in zebrafish. It is concluded that the whole extracts of $\mathrm{RAB}$ and $\mathrm{RC}$ induced angiogenesis in
\end{abstract}

Correspondence to: Dr Leung-Kim Hung, Department of Orthopaedics and Traumatology, 5/F Lui Che Woo Clinical Science Building, The Chinese University of Hong Kong, Shatin, New Territories, Hong Kong SAR, P.R. China

E-mail: leungkimhung@cuhk.edu.hk

Dr Chun-Hay Ko, Institute of Chinese Medicine, E302, Science Centre East Block, The Chinese University of Hong Kong, Shatin, New Territories, Hong Kong SAR, P.R. China

E-mail: gohey@cuhk.edu.hk

Key words: Radix Achyranthis Bidentatae, radix cyathulae, pro-angiogenesis, zebrafish, cell migration
HUVECs in vitro and in zebrafish in vivo via increasing cell migration.

\section{Introduction}

Angiogenesis is a critical physiological process responsible for forming new blood vessels from existing blood vessels (1). Endothelial cells (ECs) evidently participate in this process with several mechanisms, including proliferation, migration and assembly (2). These mechanisms are regulated by a number of angiogenic factors, including angiogenic growth factors and their receptors, transcription factors, matrix degradation molecules, cell adhesion molecules, tubule formation and morphogenesis factors, and molecules for blood vessel maturation (2). Normal physiological angiogenesis is rarely observed in adults except in the ovary and endometrium during females' reproductive life (3). In numerous pathological conditions, angiogenesis is also required to facilitate tissue repair by transferring more nutrients and oxygen to the ischemia sites, which is beneficial to treat several types of illnesses, including myocardial ischemia, osteonecrosis, bone fracture and ischemic chronic wounds such as a diabetic limb (4-6).

'Niu-Xi' is a widely used traditional Chinese medicine (TCM). Niu-Xi is traditionally believed to have the effects of removing 'blood stasis', antifertility, anti-inflammation, stimulating menstruation and curing orthopaedic diseases, including bone injury (7), although these effects have not been established clinically. As listed in the Chinese pharmacopoeia 2010, there are two different types of medicinal plants used as 'Niu-Xi' that are grown in different provinces in China. One is called Radix Achyranthis Bidentatae (RAB; named 'Huai-Niu-Xi' in Chinese), and the other one is called Radix Cyathulae (RC; called 'Chuan-Niu-Xi' in Chinese). RAB is the dried root of Achyranthese bidentata $\mathrm{Bl}$, which is grown in Africa, Asia and the He-Nan province in China. Moreover, $\mathrm{RC}$ is the dried root of Cyathula officinalis Kuan which is harvested in the Si-Chuan province in China (7). These two TCM remedies are believed to possess similar therapeutic effects on the 
above mentioned illnesses, and can be used interchangeably in different TCM formulae in numerous cases (7). In addition to these effects, use of RAB has been attempted for releasing hypertension dizziness, while RC is typically used with the aim of treating rheumatism, placenta retention and to relieve stranguria (7). The major components in these two radixes are phytoecdysones and triterpenoid saponins. The majority of them are derivatives of oleanolic acid, and have anti-inflammatory, anti-oxidant and anti-osteoporosis biological effects that have been reported $(8,9)$. However, their chemical markers are different. As suggested by the Chinese pharmacopoeia, $\beta$-ecdysterone is one of the major bioactive markers in the $\mathrm{RAB}$ extract, and cyasterone is found as an authentic standard in the RC extract $(7,10,11)$.

Although there is no direct concept on angiogenesis in the principles of TCM, RAB and RC are traditionally applied with the aim of stimulating menstruation and curing bone diseases in which angiogenesis is critical (3-5). The aim of the present study was to explore whether the whole extracts of $\mathrm{RAB}$ and $\mathrm{RC}$ both possess potential pro-angiogenic effects, which may indicate the pharmacological mechanism underlying their hypothesized traditional therapeutic effects. In the present study, the pro-angiogenic effects of the RAB and $\mathrm{RC}$ whole extracts were measured in vitro in human umbilical vein endothelial cell (HUVEC) models, where cell proliferation and migration, and tube formation were evaluated, Furthermore, an in vivo transgenic zebrafish model was also used. The underlying mechanisms were also detected by quantitative polymerase chain reaction (qPCR) for measuring angiogenesis-related gene regulation in zebrafish.

\section{Materials and methods}

Chemicals. High performance liquid chromatography (HPLC) grade acetonitrile and ethanol were purchased from Fisher Scientific (Thermo Fisher Scientific, Inc., Leicester, UK). The iScript one-step RT-PCR kit and SYBR Green reagents used were purchased from Bio-Rad Laboratories, Inc. (Hercules, CA, USA). HUVECs were provided by the American Type Culture Collection (Manassas, VA, USA). The authentic standards (purity, >95\%) of ginsenoside Ro, chikusetsusaponin IVA, $\beta$-ecdysterone and cyasterone were from Shanghai Tauto Biotech Co., Ltd. (Shanghai, China). Trypsin, fetal bovine serum (FBS), penicillin and streptomycin were supplied by Gibco (Thermo Fisher Scientific, Inc., Grand Island, NY, USA). Matrigel ${ }^{\mathrm{TM}}$ Matrix Growth Factor Reduced was bought from BD Biosciences (Franklin Lakes, NJ, USA). F12 medium, heparin, endothelial cell growth supplement (ECGS) and all other unspecified chemicals were supplied by Sigma-Aldrich (Merck KGaA, Darmstadt, Germany).

Plant material and extraction. The dried raw herbs of RAB (harvested in Henan, China) and RC (harvested in Sichuan, China) were purchased from Guangzhou Zhixin Pharmaceu tical Co., Ltd. (Guangzhou, China). Their voucher specimens (no. 2013-3413 for RAB and no. 2013-3414 for RC) were kept in the museum of the Institute of Chinese Medicine of the Chinese University of Hong Kong (Hong Kong, China) and were authenticated by thin layer chromatography (HPTLC) according to the Chinese Pharmacopoeia 2010. The presence of oleanolic acid and cyasterone were determined in RAB and $\mathrm{RC}$, using HPTLC silica gel $\mathrm{F}_{254}$ plates (Merck KGaA).

The raw materials ( $60 \mathrm{~g}$ ) of $\mathrm{RAB}$ and $\mathrm{RC}$ were extracted by 1-h heat water reflux extraction with $2 \mathrm{~L}$ of water. Following filtration, the supernatant was collected, the residue extracted by heat ethanol reflux extraction with $2 \mathrm{~L}$ of $95 \%$ ethanol for $1 \mathrm{~h}$, then filtered again to collect the ethanol. The ethanol was removed by a vacuum rotary evaporator under reduced pressure, and then mixed with the supernatant. Following overnight precipitation, the supernatant was subjected to freeze-drying in order to collect the whole extract. The dried extract was then stored in the desiccator before use.

Chemical profiles. RAB and RC whole extracts were completely dissolved in water to a concentration of $5 \mathrm{mg} / \mathrm{ml}$ and filtered with a $0.22-\mu \mathrm{m}$ filter. Next, they were subjected to chemical analysis by an Agilent 1290 Infinity HPLC system (Agilent Technologies, Inc., Santa Clara, CA, USA). HPLC was equipped with an online degasser, a binary-pump, an autosampler and a diode array detector, and an Alltima HPLC C18 column $(250 \times 4.6 \mathrm{~mm}, 5 \mu \mathrm{m}$; Fisher Scientific) protected by a C18 guard column was used for HPLC analysis according to previous reports, with minor modifications $(10,11)$. Moreover, the column was maintained at $40^{\circ} \mathrm{C}$. The mobile phase for the RAB extract consisted of (A) $0.1 \%$ acetate acid and (B) acetonitrile with the following gradient: $10-20 \% \mathrm{~B}$ from 0 to $10 \mathrm{~min}$; $20-40 \% \mathrm{~B}$ from 20 to $30 \mathrm{~min}$; $40-60 \% \mathrm{~B}$ from 30 to $40 \mathrm{~min}$; $60-90 \% \mathrm{~B}$ from 40 to $60 \mathrm{~min}$ and $90 \% \mathrm{~B}$ from 60 to $70 \mathrm{~min}$. The mobile phase for the RC extract consisted of (A) $0.1 \%$ acetate acid and (B) acetonitrile in the following gradient: $10-20 \% \mathrm{~B}$ from 0 to $10 \mathrm{~min} ; 20-28 \% \mathrm{~B}$ from 10 to $20 \mathrm{~min} ; 28 \%$ B from 20 to $40 \mathrm{~min} ; 28-60 \%$ B from 40 to $50 \mathrm{~min}$; $60-90 \% \mathrm{~B}$ from 50 to $60 \mathrm{~min}$ and $90 \% \mathrm{~B}$ from 60 to $70 \mathrm{~min}$. Moreover, the flow rate was set to $0.7 \mathrm{ml} / \mathrm{min}$ and the injection volume was $10 \mu \mathrm{l}$.

Mass spectrometry was performed using an Agilent 6530 Accurate-Mass Quadrupole Time-of-Flight mass spectrometer (Agilent Technologies, Inc.) equipped with a Jet Stream electrospray ionization (ESI; Agilent Technologies, Inc., Santa Clara, CA, USA) source. Parameters for the ESI source were set as follows: Positive ion mode; gas temperature, $350^{\circ} \mathrm{C}$; drying gas, 11 1/min; nebulizer, $55 \mathrm{psi}$; and capillary, 4,200 V. Moreover, the mass range was set to 100-1,700. Data were qualitatively analyzed using MassHunter Workstation software, version B.05.00 (Agilent Technologies, Inc.).

Cell viability. Cell viability was measured using an MTT assay. DMEM/F12 medium was mixed with heparin (100 mg/l), ECGS $(30 \mathrm{mg} / \mathrm{l})$ and $0.5 \%$ antibiotics (penicillin-streptomycin-100X, 50 mg/ml; Gibco; Thermo Fisher Scientific, Inc.). Next, the mixed DMEM/F12 medium was supplemented with $10 \%$ FBS as culture medium or $0.5 \%$ FBS as test medium. In brief, HUVECs $\left(1 \times 10^{4}\right.$ cells per well) were seeded onto the 96-well plates with culture medium overnight, and the medium was then replenished with test medium for another $12 \mathrm{~h}$. RAB and $\mathrm{RC}$ whole extract were redissolved into the stock solution $(800 \mu \mathrm{g} / \mathrm{ml})$ with test medium, filtered by a $0.22-\mu \mathrm{m}$ filter, and diluted with test medium to the working concentrations $(0-800 \mu \mathrm{g} / \mathrm{ml})$ for a $24-\mathrm{h}$ incubation. Following drug treatment, the medium was replaced with MTT $(0.5 \mathrm{mg} / \mathrm{ml}$ in test 
Table I. Cell migration-related genes (obtained from Liu et al, 2013).

\begin{tabular}{lc}
\hline Primer & Sequences \\
\hline Matrix metallopeptidase 2 & F-AGCTTTGACGATGACCGCAAATGG \\
Matrix metallopeptidase 9 & R-TCAGAATGCTCTAAACCCAGGGCA \\
& F-AACCACCGCAGACTATGACAAGGA \\
TIMP metallopeptidase inhibitor 2 & R-GTGCTTCATTGCTGTTCCGTCAA \\
& F-ATAAGCATGCGCTGAGGAAGAGGA \\
Plasminogen & R-AGCTGCAACAATCCAACTCCATGC \\
& F-CATGCAAAGGCCTTGATGGGAACT \\
Cadherin-associated protein $\beta$ ( $\beta$-catenin) & R-TTGATCTCCACAACTAGGCACGCT \\
$\beta$-actin & F- ATGAGGGCATGCAGATACCTTCCA \\
& R-TTGACCACGGCATGTTTGAGCATC \\
& F- TCCCCTTGTTCACAATAACC \\
\hline
\end{tabular}

TIMP, tissue inhibitor of metalloproteinase.

medium) for another 3 -h incubation at $37^{\circ} \mathrm{C}$, and replenished with dimethyl sulfoxide $(100 \mu \mathrm{l})$ in order to dissolve the formed formazan. The absorbance was detected at $540 \mathrm{~nm}$ using a microplate reader.

Wound healing. A wound healing assay (scratch assay) was performed as described previously (12). HUVECs $\left(1.5 \times 10^{5}\right.$ cells per well) were seeded in culture medium onto 24 -well plates overnight. Two crosses were scratched on the cells with a p200 pipette tip, then medium was removed and the cells were washed with pre-warmed PBS. Different concentrations $(0-200 \mu \mathrm{g} / \mathrm{ml})$ of $\mathrm{RAB}$ and $\mathrm{RC}$ whole extracts in test medium were prepared as mentioned above and were added for an 8 -h treatment. Images were then captured before and after drug treatment at a $\mathrm{x} 40$ magnification by an inverted microscope (Eclipse TS100; Nikon Corporation, Tokyo, Japan). Data were analyzed using TScratch software (13). Four replicates were performed for each individual experiment, and four experiments were performed.

Tube formation. RAB and RC whole extracts were redissolved into the stock solution $(800 \mu \mathrm{g} / \mathrm{ml})$ with culture medium and filtered. HUVECs $\left(1 \times 10^{4}\right.$ cells per well) were mixed with various concentrations $(0-200 \mu \mathrm{g} / \mathrm{ml})$ of $\mathrm{RAB}$ and $\mathrm{RC}$ whole extracts in culture medium, and seeded onto matrigel-pre-coated 96-well plates for a 2-h incubation. Next, the formed tube networks were monitored at a $\times 40$ magnification using an inverted microscope. The total length of tubes in each image was measured using Image-Pro Plus 6.0 software (Media Cybernetics, Inc., Rockville, MD, USA). Duplicates or triplicates were performed for each experiment, and three experiments were performed in total.

Sprout number count in zebrafish. The zebrafish TG (fli1:EGFP) ${ }^{\mathrm{y} 1} /+(\mathrm{AB})$ line was transgenic with ECs expressing enhanced green fluorescent protein, which was supplied by the Zebrafish International Resource Centre of the University of Oregon (Eugene, OR, USA). Zebrafish experiments were approved by the Department of Health, Hong Kong SAR according to the guidelines in the Care and Use of Animals. Zebrafish were sacrificed by TRIzol (Qiagen Sciences, Inc., Gaithersburg, MD, USA Briefly, zebrafish embryos at 1-4 cell stage were collected for disinfection with methyl-blue solution $(2 \mu \mathrm{g} / \mathrm{ml})$ as reported previously (12). Disinfected embryos were washed twice with embryo medium $(0.06 \mathrm{~g} / \mathrm{l}$ Instant Ocean Sea Salt, Blacksburg, VA, USA) and RAB and $\mathrm{RC}$ whole extracts were redissolved into the stock solution $(800 \mu \mathrm{g} / \mathrm{ml})$ with embryo medium, filtered by a $0.22-\mu \mathrm{m}$ filter. Next, they were diluted with embryo medium to the working concentrations. Embryos were incubated with different concentrations $(0-200 \mu \mathrm{g} / \mathrm{ml})$ of RAB and $\mathrm{RC}$ whole extracts. Moreover, embryos receiving embryo medium alone served as a control. After 72-h post-fertilization, the morphology of the sub-intestinal vessel (SIV) region in zebrafish larvae was monitored using an IX71S8F-2 inverted fluorescent microscope (Olympus Corporation, Tokyo, Japan). The mean sprout number in the SIV region was calculated by dividing the sum of the sprout number by the total number of embryos in each group, which was used for indicating the pro-angiogenic effect.

qPCR analysis of mRNA expression in zebrafish. After counting sprout numbers, zebrafish were stored in TRIzol (Qiagen Sciences, Inc.). The total RNA was extracted from whole embryos using an RNeasy Mini kit (Qiagen Sciences, Inc.) according to manufacturer's instructions. qPCR was performed with Qiagen iScript one-step RT-PCR kit and SYBR Green using a CFX96 ${ }^{\mathrm{TM}}$ Real-Time System (Bio-Rad Laboratories, Inc.). HotStarTaq DNA Polymerase (Qiagen Sciences, Inc.). The cycling conditions were set as follows: $50^{\circ} \mathrm{C}$ for $10 \mathrm{~min}, 95^{\circ} \mathrm{C}$ for $5 \mathrm{~min}$, then 50 cycles of $95^{\circ} \mathrm{C}$ for $10 \mathrm{sec}$ and $60^{\circ} \mathrm{C}$ for $30 \mathrm{sec}$. The RT-PCR primers for migration genes were synthesized by Tech Dragon Limited (Hong Kong, China) and are listed in Table I according to our previous study (12). $\beta$-actin served as a housekeeping gene. All detections were performed in triplicate, and gene expression analysis was performed to calculate their normalized expression using the $2^{-\triangle \Delta C a}$ method (14) with the CFX Manager ${ }^{\mathrm{TM}}$ software (Bio-Rad Laboratories, Inc.). 
A
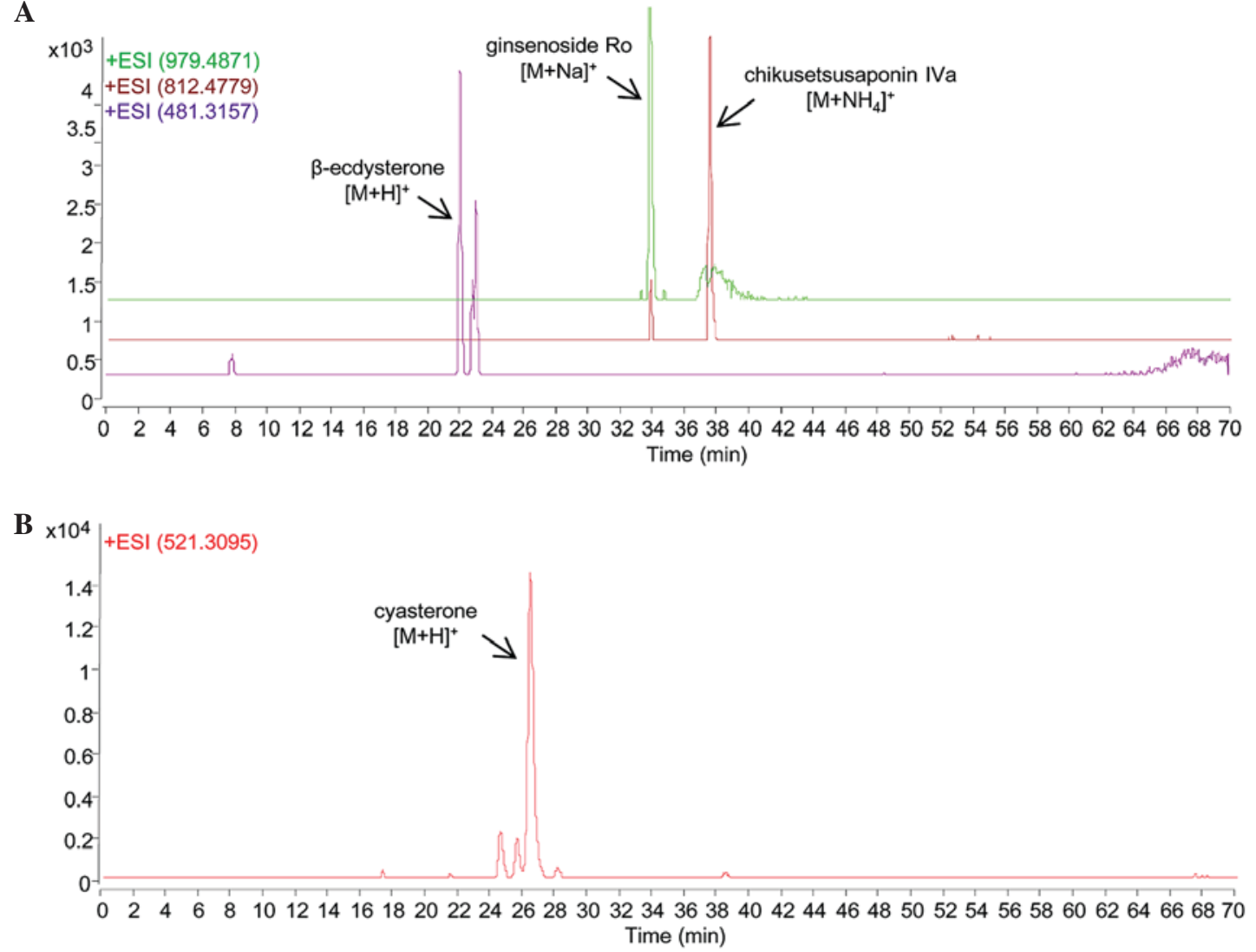

Figure 1. Representative extracted ion chromatograms of chemical markers in the whole extracts of (A) Radix Achyranthis bidentatae and (B) Radix Cyathulae. ESI, electrospray ionization.

Statistical analysis. Data are presented as the mean \pm standard error of the mean. Statistical analysis was performed by Student t-test or one-way analysis of variance followed by Dunnett's post-test and analyzed using GraphPad Prism software, version 4 (GraphPad Software, Inc., La Jolla, CA, USA). $\mathrm{P}<0.05$ was used to indicate a statistically significant difference.

\section{Results}

Chemical profiles. As shown in Fig. 1, the chemical markers in the RAB extract were identified with their respective molecular weights and retention times when compared to their authentic standards, respectively (Fig. 1A). $\beta$-ecdysterone, which is the chemical marker indicated in the Chinese pharmacopoeia for $\mathrm{RAB}$, was found with $[\mathrm{M}+\mathrm{H}]^{+} \mathrm{m} / \mathrm{z}$ at 481.3157 (error, $17 \mathrm{ppm}$ ). The other two chemical markers, namely ginsenoside Ro and chikusetsusaponin IVA, were detected with $[\mathrm{M}+\mathrm{Na}]^{+} \mathrm{m} / \mathrm{z}$ at 979.4871 (error, $0.8 \mathrm{ppm}$ ) and $\left[\mathrm{M}+\mathrm{NH}_{4}\right]^{+} \mathrm{m} / z$ at 812.4779 (error, $2.2 \mathrm{ppm})$, respectively.

Cyasterone is the chemical marker indicated in the Chinese pharmacopoeia for $\mathrm{RC}$, and it was identified in the $\mathrm{RC}$ extract with $[\mathrm{M}+\mathrm{H}]^{+} \mathrm{m} / \mathrm{z}$ at 521.3095 (error, $3.6 \mathrm{ppm}$ ) when compared with its authentic standard.

Cell viability. As detected by the MTT assay, 24-h treatment of RAB whole extract did not significantly alter the cell viability from 3.125 to $200 \mu \mathrm{g} / \mathrm{ml}$ but displayed cytotoxicity at 400 and $800 \mu \mathrm{g} / \mathrm{ml}$ when compared to the control $(\mathrm{P}<0.001)$ (Fig. 2A).
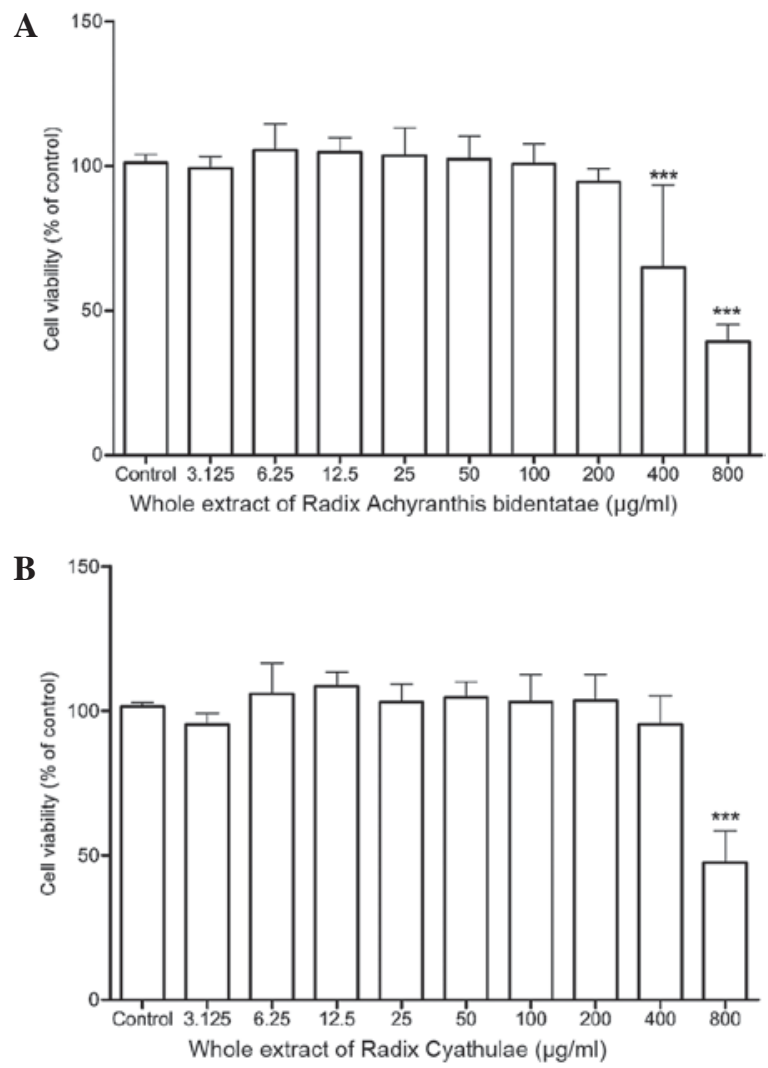

Figure 2. Effects of the whole extracts of (A) Radix Achyranthis bidentatae and (B) Radix Cyathulae on the cell viability in human umbilical vein endothelial cells in vitro, as detected by the MTT assay. Data are expressed as the mean \pm standard error of the mean $(n=3) .{ }^{* * *} \mathrm{P}<0.001$ vs. control. 


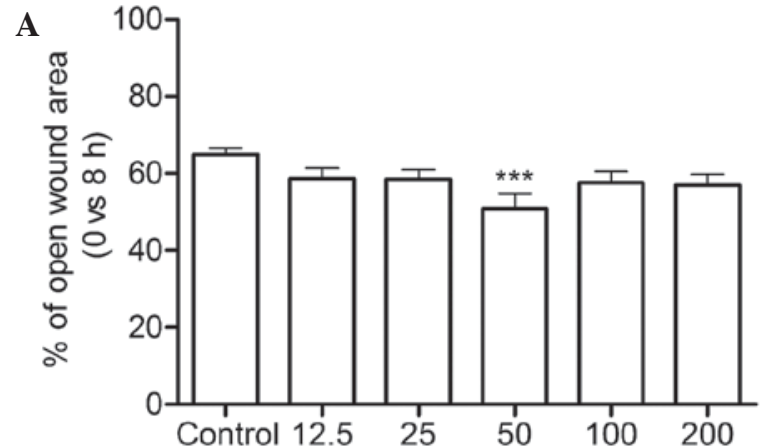

Whole extract of Radix Achyranthis bidentatae $(\mu \mathrm{g} / \mathrm{ml})$

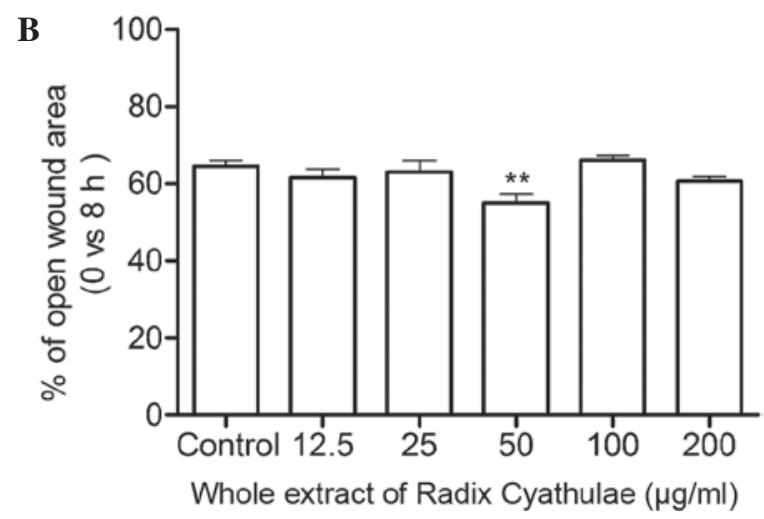

Figure 3. Whole extracts of (A) Radix Achyranthis bidentatae and (B) Radix Cyathulae enhanced cell migration in human umbilical vein endothelial cells in vitro, as detected by the scratch assay. Data are expressed as the mean \pm standard error of the mean $(n=4){ }^{* * *} \mathrm{P}<0.01$ and ${ }^{* * * *} \mathrm{P}<0.001$ vs. control.
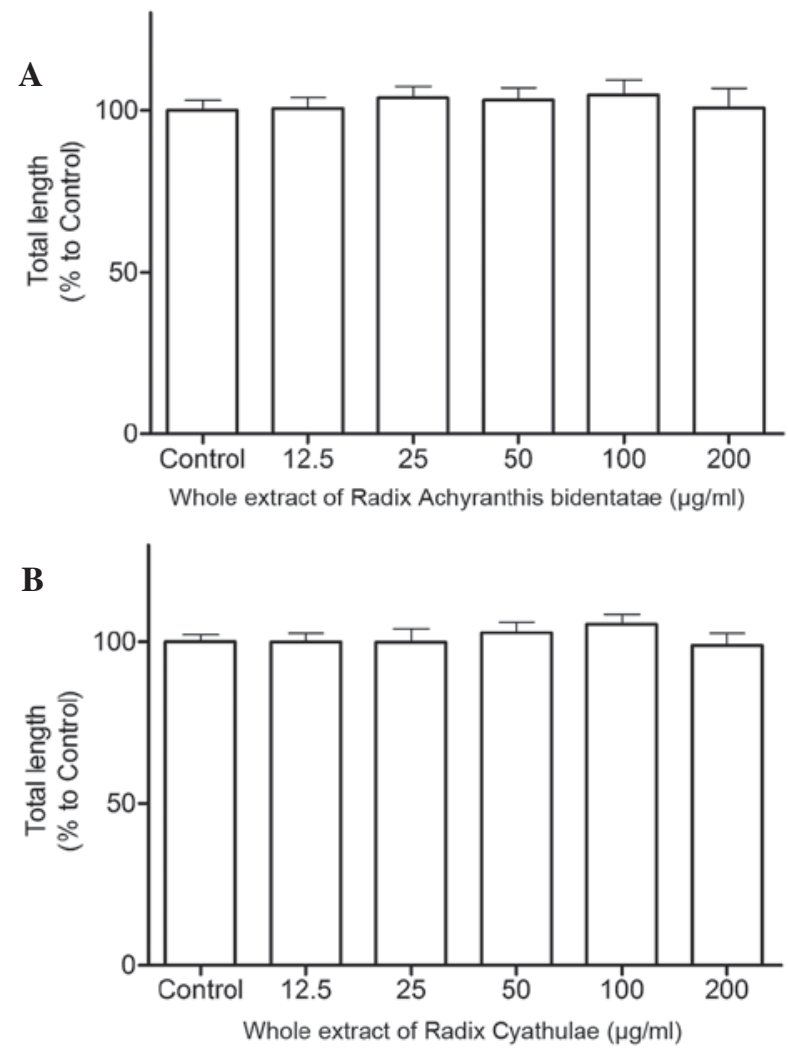

Figure 4. Whole extracts of (A) Radix Achyranthis bidentatae and (B) Radix Cyathulae did not promote tube formation in human umbilical vein endothelial cells in vitro. Data are expressed as the mean \pm standard error of the mean $(n=3)$.
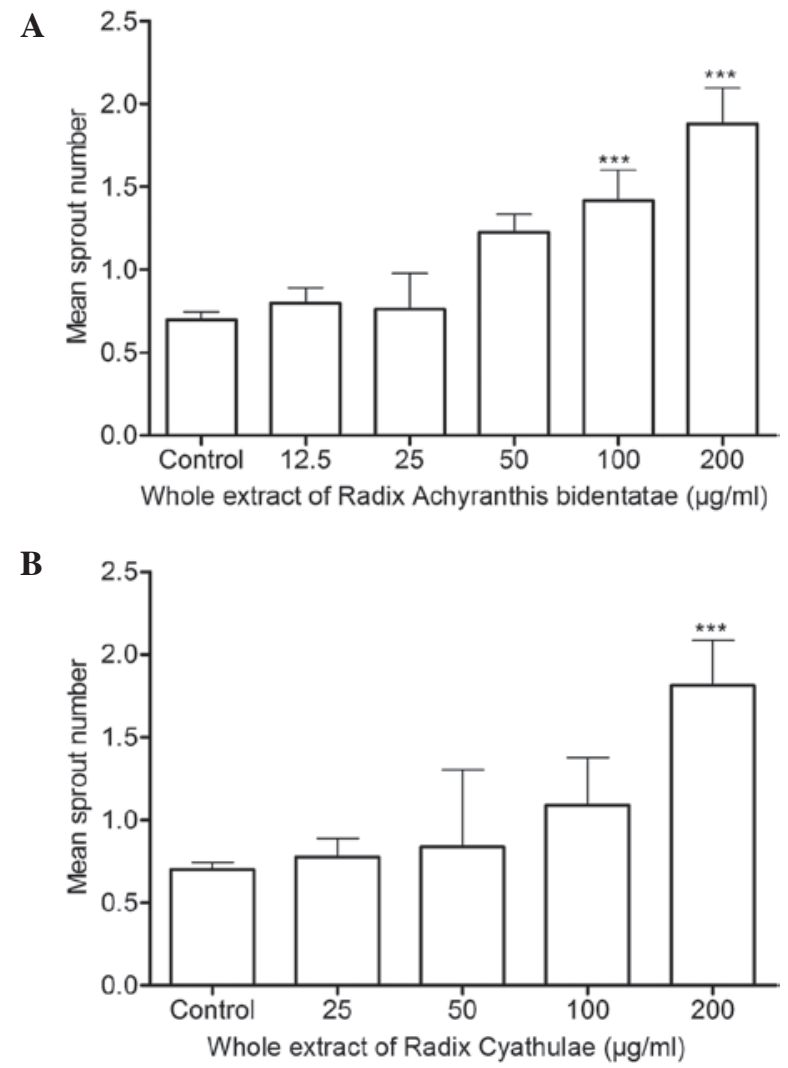

Figure 5. Whole extracts of (A) Radix Achyranthis bidentatae and (B) Radix Cyathulae increased sprout numbers in the sub-intestinal vessels in TG (fli1:EGFP) $)^{\mathrm{y} 1} /+(\mathrm{AB})$ zebrafish embryos in vivo. Data are expressed as the mean \pm standard error of the mean of three independent experiments (15-30 larvae for each experiment). ${ }^{* * *} \mathrm{P}<0.001 \mathrm{vs.} \mathrm{control.}$

Meanwhile, the RC whole extract also did not significantly increase the cell viability from 3.125 to $400 \mu \mathrm{g} / \mathrm{ml}$ but only demonstrated a significant cytotoxicity at $800 \mu \mathrm{g} / \mathrm{ml}$ (Fig. 2B).

Wound healing. As shown in Fig. 3A, the RAB whole extract significantly increased cell migration at $50 \mu \mathrm{g} / \mathrm{ml}$ after an 8 -h treatment $(\mathrm{P}<0.001)$. Moreover, the open wound area at $50 \mu \mathrm{g} / \mathrm{ml}$ was smaller than that of the control group by $\sim 21.7 \%$. In Fig. 3B, the RC whole extract evidently enhanced cell migration with a smaller open wound area by $\sim 14.9 \%$.

Tube formation. Following a 2 -h treatment, $\mathrm{RAB}$ and $\mathrm{RC}$ whole extracts at $100 \mu \mathrm{g} / \mathrm{ml}$ both individually increased tube formation in HUVECs with 5\% enhancement, but the increase was not significant compared to the control (Fig. 4).

Sprout number count in the zebrafish SIV region. When compared to the control group, RAB whole extract at 100 and $200 \mu \mathrm{g} / \mathrm{ml}$ significantly increased the sprout numbers in the SIV region of zebrafish $(\mathrm{P}<0.001)$ (Fig. 5A). Meanwhile, $\mathrm{RC}$ whole extract at $200 \mu \mathrm{g} / \mathrm{ml}$ markedly enhanced SIV sprout numbers (Fig. 5B). These indicate that RAB and RC whole extracts can both promote angiogenesis in zebrafish.

mRNA expression in zebrafish. In Fig. 6, RAB and RC whole extracts regulated migration-related genes. In detail, RAB whole extract $(200 \mu \mathrm{g} / \mathrm{ml})$ increased the gene expression of 

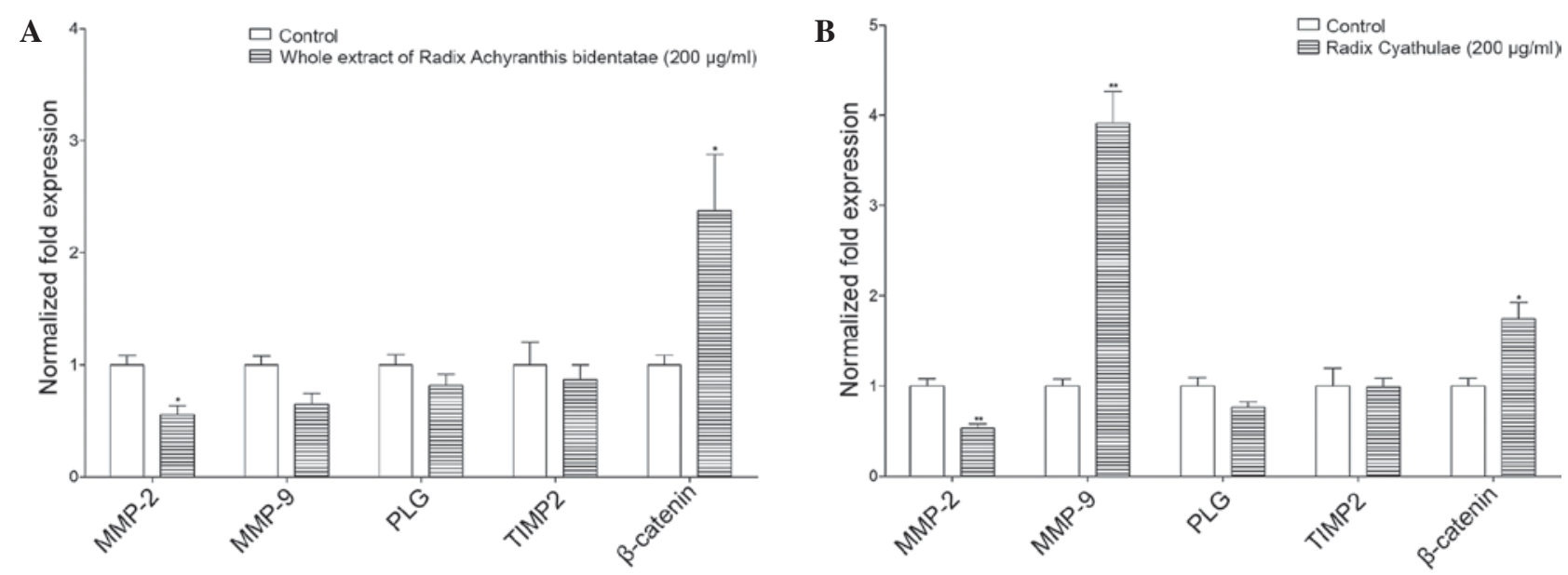

Figure 6. Whole extracts of (A) Radix Achyranthis bidentatae and (B) Radix Cyathulae regulated cell migration-related mRNA expression in zebrafish in vivo, as quantified by the quantitative polymerase chain reaction with $\beta$-actin as a housekeeping gene. Data are expressed as the mean \pm standard error of the mean of the relative mRNA amount $(\mathrm{n}=3)$. ${ }^{*} \mathrm{P}<0.05$ and ${ }^{* *} \mathrm{P}<0.01$ vs. control. MMP, matrix metallopeptidase; TIMP, tissue inhibitor of metalloproteinase; PLG, plasminogen.

$\beta$-catenin (2.4-fold) but decreased the expression of matrix metallopeptidase 2 (MMP2) by 45\%. Moreover, the expression of MMP9, plasminogen (PLG) or tissue inhibitor of metalloproteinase 2 (TIMP2) was not significantly reduced by the $\mathrm{RAB}$ whole extract.

In addition, the RC whole extract $(200 \mu \mathrm{g} / \mathrm{ml})$ significantly enhanced the gene expression of $\beta$-catenin $(1.7$-fold; $\mathrm{P}<0.05)$, but reduced the expression of MMP2 by $47 \%$. However, the $\mathrm{RC}$ whole extract did not significantly regulate the expression of PLG and TIMP2.

\section{Discussion}

Angiogenesis is important in the therapeutics used for menstruation, wound healing and bone diseases. There are several regulatory sites by exogenous compounds for stimulating angiogenesis. For example, TCM can activate multiple pathways for promoting angiogenesis (15-18). However, in the present study, the whole extracts of RAB and RC promoted angiogenesis mainly via stimulating migration of ECs in HUVECs in vitro, but not through cell proliferation or tube formation. Subsequently, several cell migration-related genes appeared to play roles in pro-angiogenesis of these two extracts in zebrafish in vivo. The results in human ECs are quite comparable to those in zebrafish.

Cell migration is one of the critical processes in angiogenesis. It highly integrates multistep processes, including cell polarization, lamellipodia formation and focal adhesions' assembly and disassembly (19). Among these steps, proteolysis and adhesion of the extracellular matrix appear to be the key steps (20). As revealed by qPCR in zebrafish, the gene expression of matrix degradation-related factor-like MMP9 and cell-cell adhesion factors such as $\beta$-catenin were upregulated. In Fig. 6, RAB and RC whole extracts regulated some of these genes, which are important in migration. In detail, the RAB extract significantly enhanced the gene expression of $\beta$-catenin but decreased that of MMP2, and very mildly reduced those of MMP9, TIMP2 and PLG. This indicates that enhancing cell-cell adhesion activity is the major process in the pro-angiogenic effect of the RAB extract. Similarly, the RC extract enhanced the gene expression of MMP9 and $\beta$-catenin, revealing that matrix degradation and cell-cell adhesion were simultaneously triggered by the RC extract for stimulating angiogenesis. As the same molecular regulator activated by both extracts, $\beta$-catenin is a critical member of the canonical Wnt signaling pathway that is responsible for vascular sprouting, particularly for cell-to-cell adhesion (21). Furthermore, it can trigger signal transduction for vascular remodeling and differentiation (22). In addition, the gene expression of MMP9 was triggered by RC but not by $\mathrm{RAB}$, indicating this as the minor difference in angiogenic mechanisms between these two extracts. Overall, the extracts of RAB and RC possess angiogenic effects through similar mechanisms, which should be one of the reasons why they can be used interchangeably in different TCM formulae for treating numerous illnesses (7).

As indicated in the Chinese Pharmacopoeia 2010, both $\mathrm{RAB}$ and $\mathrm{RC}$ are believed to treat amenorrhea and bone-related disease within the TCM system, including bone fracture healing. In the treatments of these diseases, the role of angiogenesis has been demonstrated in numerous scientific reports (3-5). For example, physiological angiogenesis always occurs in the endometrium during the menstrual cycle, and appears in the basalis layer during menstruation and in the functionalis and subepithelial capillary plexus during the proliferative and early secretory stages (3). In addition, it is well-known that blood vessel formation is associated in bone regeneration (23). During bone fracture healing, ingrowth of blood vessels are responsible for supplying nutrients and the influx of osteoblasts (24). In summary, the pro-angiogenic capacity of RAB and RC is hypothesized to be responsible for one of the therapeutic mechanisms of their traditional uses.

It is concluded that whole extracts of RAB and RC promoted angiogenesis in HUVECs in vitro and in zebrafish in vivo mainly through promoting cell migration. As mentioned above, angiogenesis is important in stimulating menstruation and bone healing. Therefore, from a modern pharmacology point of view, the present study revealed for the first time that 
pro-angiogenesis may be among the pharmacological mechanisms underlying the purported traditional therapeutic effects of $\mathrm{RAB}$ and $\mathrm{RC}$.

\section{Acknowledgements}

The present study was financially supported by the Innovation and Technology Commission, the government of Hong Kong SAR (grant no. GHX/002/11) and Alberta Technology Limited.

\section{References}

1. Polverini PJ: Angiogenesis in health and disease: Insights into basic mechanisms and therapeutic opportunities. J Dent Educ 66: 962-975, 2002.

2. Lin CM, Chiu JH, Wu IH, Wang BW, Pan CM and Chen YH: Ferulic acid augments angiogenesis via VEGF, PDGF and HIF-1 alpha. J Nutr Biochem 21: 627-633, 2010.

3. Gargett CE and Rogers PA: Human endometrial angiogenesis. Reproduction 121: 181-186, 2001.

4. Beamer B, Hettrich C and Lane J: Vascular endothelial growth factor: An essential component of angiogenesis and fracture healing. HSS J 6: 85-94, 2010.

5. Folkman J: Angiogenesis in cancer, vascular, rheumatoid and other disease. Nat Med 1: 27-31, 1995.

6. Shohet RV and Garcia JA: Keeping the engine primed: HIF factors as key regulators of cardiac metabolism and angiogenesis during ischemia. J Mol Med (Berl) 85: 1309-1315, 2007.

7. C.P. Commission, 2010. The Pharmacopoeia of the People's Republic of China version 2010. China Medical Science Press, Beijing, China.

8. Gao L, Cai G and Shi X: Beta-ecdysterone induces osteogenic differentiation in mouse mesenchymal stem cells and relieves osteoporosis. Biol Pharm Bull 31: 2245-2249, 2008.

9. Zhang X, Xu X, Xu T and Qin S: $\beta$-Ecdysterone suppresses interleukin-1 $\beta$-induced apoptosis and inflammation in rat chondrocytes via inhibition of NF-kB signaling pathway. Drug Dev Res 75: 195-201, 2014.

10. Li J, Li P, Li HJ, Song Y, Bi ZM and Li YJ: Simultaneous qualification and quantification of eight triterpenoids in radix achyranthis bidentatae by high-performance liquid chromatography with evaporative light scattering detection and mass spectrometric detection. J Sep Sci 30: 843-850, 2007.

11. Li J, Qi H, Qi LW, Yi L and Li P: Simultaneous determination of main phytoecdysones and triterpenoids in radix achyranthis bidentatae by high-performance liquid chromatography with diode array-evaporative light scattering detectors and mass spectrometry. Anal Chim Acta 596: 264-272, 2007.
12. Liu CL, Kwok HF, Cheng L, Ko CH, Wong CW, Ho TW, Leung PC, Fung KP and Lau CB: Molecular mechanisms of angiogenesis effect of active sub-fraction from root of Rehmannia glutinosa by zebrafish sprout angiogenesis-guided fractionation. J Ethnopharmacol 151: 565-575, 2014.

13. Gebäck T, Schulz MM, Koumoutsakos P and Detmar M: TScratch: A novel and simple software tool for automated analysis of monolayer wound healing assays. Biotechniques 46: 265-274, 2009.

14. Livak KJ and Schmittgen TD: Analysis of relative gene expression data using real-time quantitative PCR and the 2(-Delta Delta C(T)) Method. Methods 25: 402-408, 2001.

15. Hong SJ, Wan JB, Zhang Y, Hu G, Lin HC, Seto SW, Kwan YW, Lin ZX, Wang YT and Lee SM: Angiogenic effect of saponin extract from Panax notoginseng on HUVECs in vitro and zebrafish in vivo. Phytother Res 23: 677-686, 2009.

16. Lam HW, Lin HC, Lao SC, Gao JL, Hong SJ, Leong CW, Yue PY, Kwan YW, Leung AY, Wang YT and Lee SM: The angiogenic effects of Angelica sinensis extract on HUVEC in vitro and zebrafish in vivo. J Cell Biochem 103: 195-211, 2008.

17. Li S, Lou S, Lei BU, Chan TF, Kwan YW, Chan SW, Leung GP, Tsui SK and Lee SM: Transcriptional profiling of angiogenesis activities of calycosin in zebrafish. Mol Biosyst 7: 3112-3121, 2011.

18. Zhou X, Siu WS, Fung CH, Cheng L, Wong CW, Zhang C, Liu CL, Kwok HF, Lau CP, Wat E, et al: Pro-angiogenic effects of Carthami Flos whole extract in human microvascular endothelial cells in vitro and in zebrafish in vivo. Phytomedicine 21: 1256-1263, 2014.

19. Ridley AJ, Schwartz MA, Burridge K, Firtel RA, Ginsberg MH, Borisy G, Parsons JT and Horwitz AR: Cell migration: Integrating signals from front to back. Science 302: 1704-1709, 2003.

20. Gontero P, Banisadr S, Frea B and Brausi M: Metastasis markers in bladder cancer: A review of the literature and clinical considerations. Eur Urol 46: 296-311, 2004.

21. Liebner S, Cavallaro U and Dejana E: The multiple languages of endothelial cell-to-cell communication. Arterioscler Thromb Vasc Biol 26: 1431-1438, 2006.

22. Reis M and Liebner S: Wnt signaling in the vasculature. Exp Cell Res 319: 1317-1323, 2013.

23. Sun H, Jung Y, Shiozawa Y, Taichman RS and Krebsbach PH: Erythropoietin modulates the structure of bone morphogenetic protein 2-engineered cranial bone. Tissue Eng Part A 18: 2095-2105, 2012.

24. Khosla S, Westendorf JJ and Mödder UI: Concise review: Insights from normal bone remodeling and stem cell-based therapies for bone repair. Stem Cells 28: 2124-2128, 2010. 